\title{
Evaluation of the Medical Diagnostic Imaging Support System Based on 2 Years of Clinical Experience
}

\author{
D.V. Smith, S. Smith, G.N. Bender, J.R. Carter, Y. Kim, M.A. Cawthon, \\ R.G. Leckie, J.C. Weiser, J. Romlein, and F. Goeringer
}

The Medical Diagnostic Imaging Support (MDIS) system at Madigan Army Medical Center (MAMC) has been operational in a phased approach since March 1992. Since then, nearly all image acquisition has been digital with progressively increasing primary softcopy diagnosis used. More than 375,000 computed radiography (CR) images as well as other modality images have been archived. Considerable experience in installation and implementation phasing has been gained. The location and ergonomic aspects of equipment placement were refined with time. The original clinical scenario was insufficiently detailed and additions were made to facilitate smoother and more complete transition toward a filmless environment. The MDIS system effectiveness and performance have been good in terms of operational workload throughput, background operations, and reliability. The important areas regarding reliability are image acquisition, output, display, database operations, storage, and the local area network. Fail-safe strategies have been continually improved to maintain continuous clinical image availability during the times when the MDIS system or components malfunction. Many invaluable lessons have been learned for effective quality assurance in a hospital-wide picture archiving and communication system. These issues include training, operational quality control, practical aspects of CR image quality, and increased timeliness in the generation and distribution of radiographic reports. Clinical acceptability has been a continuous process as each phase has been implemented. Clinical physicians quickly used the workstations soon after the start of MDIS at MAMC. The major advantage for clinicians has been the amount of time saved when retrieving multimodality images for review. On the other hand, the radiologists have been slower in their acceptance of the workstation for routine use. Radiologists need the completed software and hardware implementation to achieve the throughput necessary for a high-volume practice setting in making primary softcopy diagnoses.

This is a US government work. There are no restrictions on its use.

KEY WORDS: filmless hospital, Medical Diagnostic Imaging Support (MDIS) System, picture archiving and communication system (PACS).

$\mathbf{T}^{\mathrm{H}}$ HE MEDICAL Diagnostic Imaging Support (MDIS) system contract for the US Department of Defense was awarded to Loral Corporation in September 1991 after a competitive bidding process for installation of multiple picture archiving and communication system
(PACS) and teleradiology sites. ${ }^{1-4}$ Madigan Army Medical Center (MAMC), the first of these sites, began installation for the initial phase immediately after the contract award, with clinical operations starting in March 1992. The goal is $90 \%$ to $95 \%$ filmless operations (excluding mammography) when implementation is complete.

MAMC is the US Army's newest tertiary care center with more than 400 inpatient beds and a large outpatient clinic facility. More than one million outpatient visits and nearly 160,000 radiologic procedures were completed in 1993. There are 14 staff radiologists and 20 diagnostic radiology residents out of the 400 total physicians at MAMC.

The present phased installation of the MDIS System at MAMC includes computed radiographs (CRs) for all plain radiographs except mammography, several digital modality interfaces, a 40-Gbyte working storage unit (WSU), a 100-platter (1-Tbyte) Kodak 6800 Optical Disk Jukebox (ODJ; Eastman-Kodak, Rochester, NY), and 25 workstations. The CR images are processed by two Fuji 7000 (Siemens Digiscan) and three Fuji AC-1+ readers.

The high-performance central image file server, the WSU, is a redundant array of inexpensive disks (level 2 architecture). ${ }^{5}$ It has a

From the Department of Radiology, Madigan Army Medical Center, Tacoma, WA; the Department of Electrical Engineering, University of Washington, Seattle; the Department of Radiology, Brooke Ammy Medical Center, Ft Sam Houston, TX; and the US Amy Medical Material Agency, Medical Diagnostic Imaging Support Project, Fort Detrick, Frederick, $M D$

The opinions or assertions contained in this article are the private views of the authors and are not to be construed as official or as reflecting the views of the Army Medical Department, the Department of the Army, or the Department of Defense.

Address reprint requests to Donald V. Smith, MD, Department of Radiology, Madigan Army Medical Center, Tacoma, WA $98431-5000$.

This is a US government work. There are no restrictions on its use.

0897-1889/95/0801-0001\$3.00/0 
proprietary backplane of $320 \mathrm{Mbits} / \mathrm{s}$ and 28 input/output ports ${ }^{6}$ and was originally developed for a US Air Force reconnaissance aircraft program. The WSU functions as local and short-term storage ${ }^{2}$ using 40 disks (magnetic media) operating in parallel: 32 disks for 32-bit words, 7 disks for error correction, and one disk acting as a "hot spare" (single disk failure detected and corrected without loss of data and availability). The WSU is designed to hold inpatient images for the average length of a hospital stay (4.5 days for MAMC), all outpatient images for 48 hours, all studies not yet interpreted, and pertinent historical images. Presently, all images obtained within the last 10 days are available on the WSU. Images are stored in the WSU with $\approx 2.5: 1$ lossless compression giving an effective storage size of 80 Gbytes.

The WSU is connected to the workstations by a fiber-optic connection in a branching star topology. Compressed image data moves at 100 Mbits $/ \mathrm{s}^{7}$ with decompression at local workstations. Newly acquired images are sent to the WSU and then archived in the ODJ at the earliest possible opportunity. The ODJ holds one hundred 10-Gbyte write-once-read-many 14-inch optical disks. CR images are stored in the ODJ after 10:1 lossy compression via an improved Joint Photographic Experts Grouplike algorithm.

The final phase calls for two ODJs which could store more than 5 years of images on line. Presently, as part of the phased implementation, all CR and computed tomography (CT) images are stored in both softcopy and hardcopy (laser-printed film) form. The digital-spot imaging (DSI) fluoroscopy examinations are softcopy only. In the near future, magnetic resonance (MR), nuclear medicine (NM), ultrasound (US), angiography, and the cardiac catheterization lab will be connected to the system. Furthermore, more than 90 additional workstations are scheduled to be placed throughout the clinics and wards of the hospital. MAMC is expected to proceed to filmless operations after installation of the additional workstations.

\section{INSTALLATION}

Physical installation of the MDIS System at MAMC required modifications from the original plan. ${ }^{8}$ This was primarily caused by the need for increased heating, ventilation, and air conditioning (HVAC) capacity. Even though
MAMC was a new facility, it was not originally designed for a PACS system. ${ }^{9}$ Each $\mathrm{CR}$ reader and dedicated printer produces $\sim 24,000 \mathrm{BTU} / \mathrm{h}$. The placement of four CR readers in two main radiology locations required 8 tons of additional HVAC capacity, which was accomplished by adding four ceiling-mounted cooling units. An alternate approach available now is to place the CR printers via local area network in an area where HVAC can be concentrated in one location or is already sufficient. Originally, only a single air handler was installed in the computer room working from one of six chillers in the hospital engineering plant. The first 10 months of operation led to over three cumulative days of downtime caused by HVAC failure in the MDIS computer room. This was the major reason for the $1.3 \%$ operational downtime experienced.${ }^{10}$ Crossover chiller capacity was possible, but required an engineer to come to the hospital during nights or weekends resulting in a delay. A dedicated redundant air handler and chiller was installed by the summer of 1993 with rapid, automatic switch when necessary. No additional HVAC-related downtime has occurred since the backup system was installed.

\section{IMPLEMENTATION PHASING}

The important global implementation issues of the MDIS System at MAMC have been software and hardware maturity, archive loading, image compression, prefetching, and digital modality integration.

\section{Software and Hardware Maturity}

The MDIS System implementation at MAMC was scheduled for a 30 -month installation as the first site. This was to ensure as smooth a transition as possible in a high-volume clinical environment. ${ }^{1,2}$ The first 6 months involved installation of the initial computer room equipment, fiber-optic cabling, and workstation placement. The subsequent 24 months has allowed for incremental delivery of software and hardware to accommodate an increasingly filmless environment. It is anticipated that in the future, large MDIS installations could reach full implementation taking much less than 30 months.

\section{Archive Loading}

Archive loading is the process of filling the long-term archive with historical images. ${ }^{8}$ In our high-volume practices as well as future MDIS system sites, immediate full softcopy operations are not possible, mostly because of the logistics of historical image digitization. There are $\sim 3$ million images in a typical military medical center's 5-year film library. The digitization of all the pertinent historical images with film 
digitizers was not deemed cost effective or practical. Instead, we have adopted a hybrid approach where all new examinations are acquired digitally with more limited use of the labor-intensive film digitization. ${ }^{8}$ Parallel hardcopy and softcopy archives are being maintained until a filmless environment can be achieved.

\section{Image Compression}

The decision to use a nominal 10:1 lossy compression for CR images for archiving after the primary diagnosis has been made was necessary to be cost effective with the long-term archive technologies available when the request for proposal was originally developed in $1990 .{ }^{11}$ The radiology literature has generally supported the view that the significant diagnostic information is not lost with this level of compression. ${ }^{12-14}$ The lossy compressed images are used for reference comparison along with the finished report. Newly acquired images are used in diagnosis with only lossless compression. The practical day-to-day observation is that the 10:1 compressed CR images have the same quality as those viewed with lossless compression. A largescale receiver-operating characteristic study in cooperation with the University of Washington and other MDIS sites is underway at MAMC to evaluate the effect of the 10:1 image compression technique used in MDIS. ${ }^{15}$

\section{Intelligent Prefetch}

The retrieval of historical images from the long-term optical archive is a critical issue for success of any PACS. ${ }^{16,17}$ Current sustained rates of dearchiving image data from the ODJ for our system is approximately one average CR examination (2.2 images) per minute with one ODJ installed. The major rate-limiting step is the image compressor/expander rather than the $\mathrm{read} /$ write performance of the jukebox. The compressor/expander archives a 10-Mbyte CR image and reduces it to 1-Mbyte data for storage on an ODJ disk and vice versa. Expected system-data retrieval performance with an improved compressor/expander and two installed jukeboxes is about five average CR examinations per minute ( 11 images $/ \mathrm{min}$ ).

Requirements to retrieve archived images not only exist for comparison with new examina- tions, but just as importantly are needed for review of patient studies at follow-up clinical appointments. Review of images with reports by clinicians is a common practice at our facility. Seventy-five percent of the radiology workload for a typical military medical center are outpatient studies. If retrieval of these examinations by the clinicians occurred on an ad hoc basis during the day, the archive would be overwhelmed. ${ }^{17}$ Rather, the clinic appointment list must be made available to the MDIS System through a hospital information system (HIS)/ radiology information system (RIS) interface. Pertinent examinations can then be dearchived during the night using clinic specific rules since retrieving all images for each patient would inundate the WSU as well. Only recent images are needed in most cases with the clinical specialty determining the specific examination choices (eg, chest examinations for the pulmonary clinic). Another important function of this $\mathrm{HIS} / \mathrm{RIS}$ interface is to trigger the long-term archive to retrieve pertinent historical images for scheduled radiology studies as well as for walk-in patients. This enables immediate softcopy diagnosis when the current study is completed.

\section{Digital Modality Integration}

One of the difficult installation issues of a PACS is integrating multiple modalities from different commercial vendors. Version 2.0 of the ACR-NEMA (American College of Radiology-National Electrical Manufacturers Association) standard was embraced by the MDIS System contract to allow direct digital transfer of imagery for CT, MR, US, DSI, NM, and angiography and cardiac catheterization labs where possible in a multi-vendor setting. ${ }^{2}$ In implementation, the standard still had many areas where conformance was vague and at times incomplete. ${ }^{18,19}$ Therefore, even when two vendors produced ACR-NEMA-compliant equipment, they could not be easily connected.

The ACR-NEMA logically compatible interface did not work well for CT until approximately 18 months into clinical operations. Interface problems included an inability to send the CT scout slice localizer overlays and reconstructed images, difficulty in linking the present examination with prior examinations in the 
patient folder, and incomplete or duplicate transmission of images in the same examination. Today, CT images are automatically sent across the network to the correct electronic patient folder.

The new DICOM (Digital Imaging and Communications in Medicine) version has removed many of the ambiguities and is more complete. However, different digital formats within the DICOM standard can still be selected. Equipment vendors need to specify the digital format that their devices support to be truly compatible. This can be done through a user conformance profile. ${ }^{20}$ Integration of different digital modalities and active support of the DICOM standard by commercial vendors are essential for the proliferation of PACS.

\section{CLINICAL SCENARIO REFINEMENT}

Different methods are available to design and implement a PACS system. ${ }^{21}$ The MDIS System's approach is to use a performance specification based on a detailed clinical scenario of hospital-wide operations related to imagery and diagnostic reports. ${ }^{2,22}$ Refinement of the clinical scenario has continued over time resulting in modifications in implementation.

\section{Workstation Distribution}

Distribution of clinical workstations depends on peak clinic activity, average length of image review, geographical layout of the clinic, and location of conference rooms. ${ }^{8,10}$ During the initial phase of the MDIS system implementation in the new MAMC hospital, a greater understanding of the operational changes that would occur with full implementation was realized, which resulted in an increase of 18 workstations for various clinics.

Eight additional workstations were added to the radiology department to adjust for changes since the original plan. The original concept design included a CR acquisition workstation that would serve to acquire $\mathrm{CR}$ images and provide basic image quality control and manipulation (image orientation, window/level changes) before releasing the examination for interpretation/viewing. This CR workstation does not have sufficient handling throughput to perform image acquisition and quality-control activities simultaneously. Thus, the two additional work- stations for quality control were placed in the two primary $\mathrm{CR}$ work areas.

Another workstation was needed for making hardcopy images. The original clinical scenario did not sufficiently address this need. The required volume of hardcopy reprints is relatively large over time because of turnover of activeduty personnel and their families to other installations, most of which do not have an MDIS system installed so that transfer of hardcopy historical images is necessary. This workstation is placed near the network laser film printer for greater throughput efficiency. The remaining five additional workstations in the radiology department are provided for increased diagnostic and review activities, largely because of the growth of MAMC's radiology residency program. These changes bring the total number of workstations for MAMC to 118, which results in an approximate ratio of 3.4 physicians per workstation. Of course, different areas and departments have varying ratios..$^{8,10}$

\section{Laser-Film Printer}

The laser-film printer was originally placed in the file room. A better location would have been near the $C R$ reader in the main work core where emergency room and after-hours inpatient examinations are processed. Since installation of the MDIS System, access to the file room is difficult after hours, and it is physically separate from the main work area. We have left the printer in the file room for now because of relatively high relocation costs.

\section{RIS Issues}

RIS functionality is essential for a successful PACS. This is provided where available by the Department of Defense's Composite Health Care System (CHCS). ${ }^{2}$ CHCS supplies an integrated approach to HIS/RIS functionality and is in the process of being interfaced to the MDIS System. A MDIS System's own interim RIS (IRIS) is being used until then.

The IRIS is not intended to duplicate CHCS and, as such, has relatively limited capability. Obtaining approval of dictated reports in the IRIS is awkward when staff members or residents leave for meetings, educational rotations, or vacations. Our solution is to have a surrogate approver. However, for each case, the system 
administrator must program the privilege to the surrogate with their name appended to the report along with the dictating radiologist's name. The other report-approval issue is the time needed to display the next report for approval on the workstation or IRIS terminal. This was up to 30 seconds initially and currently is routinely less than 10 seconds, still slow for sufficient clinical acceptance. On the other hand, CHCS will allow surrogate report approval and have less than 2-second display times for reports.

\section{MDIS SYSTEM PERFORMANCE}

The overall MDIS System effectiveness is a function of operational throughput, system reliability, and fail-safe strategies to ensure continuous clinical availability. ${ }^{23} \mathrm{~A}$ hospital operates 24 hours per day and any interruption of clinical services could have life or death consequences. The past experience of others found even a $95 \%$ system availability to be barely sufficient for user acceptance. ${ }^{24}$ The MDIS System contract system requires a $99 \%$ system availability with detailed plans for crisis management and operations for times when the system is down. Lessons have progressively been learned in these areas.

\section{Operational Throughput}

Operational throughput of image production most importantly involves CR. The number of CR readers needed is based upon peak imaging needs for each operational area. ${ }^{8}$ The main radiology department, with 14 exposure and 4 fluoroscopy rooms could theoretically produce about 200 CR images per hour at peak operations, resulting in four $\mathrm{CR}$ readers; two AC-1+ (40 plates/h) and two Fuji 7000 units (60 plates $/ h$ ). Actual measured throughput rates for CR readers are 55 plates per hour for the Fuji 7000 and 34 plates per hour for the AC-1+. There is one more AC-1+ CR in the orthopedic clinic. Multiple devices are valuable for redundancy and continuous operations when one or more CR readers are down. Actual peak loads at MAMC in main radiology have been up to 90 CR plates per hour. Excess capacity is important for full mobilization in the event of a natural disaster or war.

\section{System and Component Reliability}

Full-system and component reliability has continued to show a high degree of dependability at MAMC. ${ }^{25}$ The MDIS system continues to operate at a comparable level to the previously reported $99.7 \%$ initial system uptime (excluding HVAC-related downtime). ${ }^{10}$ The MDIS system's contractual reliability specification is very detailed to fulfill precise requirements for acceptance testing and warranty issues. ${ }^{26,27}$ Discussion here focuses on a more practical measurement of reliability; whether images can be acquired and displayed on workstations for diagnosis and review. Data collected on system and component reliability are based on a 16hour day, 7 days a week as required by the MDIS system specification. ${ }^{2,27}$ The most significant reliability issues involve the central image file server, system host processor, long-term archive, and CR readers. Scheduled system upgrades will be discussed as a separate issue and are not counted against reliability figures below.

The WSU functioning as the central image file server is the heart of the MDIS system. Mean time between failure for loss of data on this device is rated to be greater than 15 years. The WSU has been an extremely reliable component of the MDIS system. In 1993, it had an uptime record of $99.97 \%$. The very limited downtime resulted from a loose network card in the WSU and no data was lost in this episode.

A MicroVAX 4000/300 (Digital Equipment Corp, Maynard, MA) operates as the system host processor and stores the IRIS data. ${ }^{6}$ Data is mirrored on two 1-Gbyte hard drives providing some redundancy against information loss. The MicroVAX is a potential single point of failure for the MDIS System. The total MicroVAX uptime for 1993 was $99.24 \%$. One major event for unplanned downtime was database failure, caused by human error. This caused more than 30 hours of downtime while the database was reconstructed from backup tapes.

The long-term archive is presently another potential single point of failure because the second ODJ has not been installed yet. When the ODJ is down, operations can continue until the WSU fills up with new images that have not yet been archived. The problem, of course, is that the older examinations cannot be retrieved. 
Fortunately, the old radiologic reports on these examinations are available on the workstation. The ODJ was operational for $96.75 \%$ in 1993 with a majority of the downtime associated with replacement of the failed robotic arm assembly, not a part of the on-site spares.

$C R$ readers have shown a high degree of durability. ${ }^{25}$ Approximately 375,000 CR images have been processed in 29 months of continuous operations with relatively little downtime for individual units. The three AC-1 + CR readers have been operational $99.1 \%$ to $99.7 \%$ in 1993 . The single greatest cause for unavailability was a failed heater element used for warming the chemicals in the attached dedicated processor. We now carry this item in our spare parts inventory after several episodes of failure required obtaining off-site replacement. The two Fuji $7000 \mathrm{CR}$ readers process the bulk of images with an accordingly higher rate of failure. They were operational $96.7 \%$ and $98.7 \%$ in 1993 . A variety of causes were responsible for the downtime, but, in general, earlier reasons were related more to hardware (eg, jammed image plates), whereas later problems had more to do with increasingly complex software (eg, deadlocks).

Scheduled system upgrades for hardware and software have been the greatest source of substantial system downtime. Five upgrades between November 1992 and November 1993 took from 2 to 7 days depending on complexity involved. This caused the MDIS system to be only partially available for 18 days because of upgrades in this 12 -month period. Stand-alone fault tolerant strategies described below were used during these episodes. Future system upgrades should occur less frequently and take less time ameliorating this problem.

\section{Fail-safe Strategies}

Complicated electronic systems inherently have component failures. Continuous operations depend on a fault tolerant design for hardware and software. ${ }^{23}$ The most significant fail-safe strategies planned and refined for the MDIS system involve using an adjunctive miniPACS concept, dedicated modality hardcopy printers, fiber-optic network design, emergency power (including uninterruptible power supply
[UPS]), multiple ODJ, the IRIS, and database backups.

One way to improve reliability of the MDIS system is adding mini-PACS capability to each modality where possible. ${ }^{28}$ This allows for continued stand-alone operations when the full system is unavailable. Mini-PACS capability was added to the gastrointestinal (GI), NM, and US sections. The CT, MR, and digital angiography areas essentially have inherent mini-PACS with their small optical-disk backups and own workstations.

The ability to produce hardcopy images for all modalities whenever necessary is an intrinsic fail-safe strategy necessary for any PACS. Direct hardcopy printing is available for $\mathrm{CR}, \mathrm{CT}$, US, MR, NM, and digital angiography. However, one area without direct hardcopy ability is DSI. Spot images can only be stored in semiconductor memory; any power surge or failure could lose images not yet transferred to the WSU. This has resulted in restricted use of fluoroscopy when the MDIS system is down. This situation has improved since an intermediate staging workstation was installed with a hard drive to store DSI images to prevent image loss and allow less restricted use during system downtime.

Dedicated CR hardcopy printers not only allow parallel hardcopy and softcopy operations to build up the long-term archive, but also provide important image backup operations. During system upgrade periods, for instance, two hardcopy CR images are produced for each view. One copy is used clinically for diagnosis and review, while the second one is held to be digitized into the MDIS system when the upgrade is complete. The effort to find all images produced if only one copy had been made would be logistically difficult and many images would be unaccountable just because of normal clinical activity. A less disruptive, more efficient method of upgrades would involve digital spooling of the CR image data to a local storage device providing easy direct transfer of images to the MDIS system when available.

The fiber-optic network was designed to have an overlapping distribution. The network topology for the MDIS system is a branching star. ${ }^{6}$ The branch junctions are network hubs vulner- 
able to a single point of failure. Supplying clinics with network connections for their workstations from several different hubs increases the probability for nonstop clinical operations, although at reduced capability if a failure occurs. Another strategy is to install $25 \%$ additional fiber-optic connections within the hospital's interstitial spaces allowing for future needs and attrition of fibers. The cost of additional materials compared with later outlay for labor with future installation was negligible.

The UPS for the MDIS system computer room prevents corruption of the database with acute power loss. The UPS is essentially a large battery with enough reserve power to allow a graceful shutdown of the MDIS System if the backup hospital generator fails. The UPS also has the role of power conditioning and preventing power surges from reaching computer room equipment. The hospital emergency generator is tested every week with a noticeable power surge, yet this has not caused any significant problems in the MDIS system computer room. However, the CR readers with no UPS attached to them must be quiescent during emergency power switch tests. The scheduled time to start the emergency generator test was late on four occasions in 1993 causing 15 to 150 minutes of CR downtime for each episode.

The integrated fault tolerant design must include plans for power outages. Emergency generators at MAMC provide a significant amount of backup power for the hospital, but cannot keep everything operational. Critical devices must be identified and prioritized. It is not possible to keep the MDIS system fully operational in this situation. The computer room, $\mathrm{CR}$ readers in main radiology, and selected workstations are provided with continuous power. CR readers go to hardcopy print mode to support basic imaging needs during power emergencies. This scenario has worked fairly well during two major regional power outages resulting from massive storms. Even though the use of two ODJs has more to do with the need for image dearchiving rates than faulttolerant design, they will provide limited redundancy to allow archiving/dearchiving to continue if a component malfunctions on one.

CHCS will be the primary vehicle for RIS operations of the MDIS system while the IRIS functions as the backup for CHCS when it is down or as a small-scale RIS where CHCS is not installed. This will be facilitated with a bidirectional interface to allow database reconciliation when CHCS is back on-line. ${ }^{2}$ Currently, CHCS has been installed at MAMC and the CHCS interface to the MDIS system is going through various levels of testing. Database backups are performed twice a day for the most recent system activity. They typically take 20 to 30 minutes and cause some system performance degradation. These are done during nonpeak periods whenever possible to minimize impact on clinical operations.

\section{QUALITY ASSURANCE}

Quality assurance for diagnostic radiology represents all activities performed to generate a timely and accurate diagnosis. A PACS adds new dimensions to this process. The most important quality-assurance issues in implementation of the MDIS system at MAMC are training, image quality control, and finished diagnostic reports. ${ }^{10}$

\section{Training}

The use of the PACS workstation is the most important training issue. Different approaches are required for clinicians and radiologists. Turnover of personnel at military facilities is relatively high, adding an extra burden for training. Requirements have been established for new personnel to attend introductory training classes before receiving access to the system. This class is given by vendor-supplied computer technicians/trainers in a small group setting with hands-on approach. This gives them basic understanding of the MDIS system and workstation functions, but the full utilization of the workstation integrated into routine clinical practice takes more time and experience. Clinical integration has accelerated as the entire hospital staff has become familiar with use of the workstation and newcomers now have ubiquitous help from other users when questions arise.

The most important fact that clinicians must learn is the difference between $1-\mathrm{K}$ and $2-\mathrm{K}$ monitor workstations. The MDIS system supplies complete 2-K CR data sets to all worksta- 
tions, but the images on a $1-\mathrm{K}$ monitor must be magnified or zoomed to see the full 2-K information available. ${ }^{29}$ We have observed greater difficulty recognizing a pneumothorax or subtle fracture unless these functions are used on $1-\mathrm{K}$ monitors. This fact is now being emphasized in training and is less of a problem now. Certification and credentialing of physicians to use PACS workstations may eventually be necessary for risk management depending on the evolving medicolegal philosophy related to filmless medical operations. New radiologists must learn how to read a large number of examinations on the workstation. This requires training with more in-depth coverage on those topics including additional functionality, especially in the area of presets, worklists, and reporting.

\section{Image Quality Control}

Image quality control in a PACS has two major segments, hardcopy versus softcopy. Hardcopy issues are discussed first, primarily concerning CR. CR has similarities with film/screen radiography, but also has significant differences in areas of processing algorithms, dynamic range, collimation, and spatial resolution. ${ }^{30}$ The MDIS system primarily uses Fuji CR devices (Fuji 7000 and $\mathrm{AC}-1+$ ). Many of the original preset display algorithms did not work well for hardcopy images using the one-on-one format at MAMC, especially for musculoskeletal radiography. Calibrating the $\mathrm{CR}$ readers and printers ${ }^{33}$ along with changing the original algorithms improved hardcopy image quality allowing for widespread clinical acceptance.

With CR, production of image optical densities in the correct range over a wide set of exposures is possible. Consistent dose-independent optical density for hardcopy images is an advantage of CR. This has been most valuable for portable chest radiographs. ${ }^{32}$ However, problems can occur with image quantum mottle or excess patient exposure dose. With film/screen systems, a natural feedback mechanism exists in that an underexposed film is too light whereas an overexposed film is too dark. This feedback is absent in CR. Markedly underexposed images could have the correct optical density. The image is grainy because of quantum mottle that can decrease the conspicuity of subtle pathology. Many of these images passed our quality- control step initially, but this has been corrected and occurs infrequently now.

An overexposed CR plate gives an excellent image, but also an increased dose to the patient. The technologists began to gradually use a greater average dose over time because they were guaranteed a good image without any obvious penalty. Fuji's CR sensitivity numbers roughly inversely correlate with patient dose, allowing a method to follow individual and departmental trends in patient radiation exposure. The quality-control technologists now monitor the sensitivity number on all images. Regular in-service training sessions along with immediate daily feedback to technologists have reduced this tendency to increase the patient dose over time and have helped identify the images with quantum mottle.

An unexpected problem was the more precise collimation required with $\mathrm{CR}$ compared with plain radiography. The problems that MAMC has encountered mostly relate to tight or overlapping collimation with resultant hardcopy images having optical densities that are too light or too dark. Initial hardcopy repeat rates were $\sim 15 \%$. An investigation into this led to relatively quick solutions that have been passed on to the other MDIS system sites. Repeat rates more recently average $\sim 3.5 \%$, virtually all because of incorrect anatomic positioning rather than collimation or algorithm selection issues.

Although the contrast resolution of CR is superior, film/screen systems have a higher spatial resolution. The spatial resolution of CR ranges from 2.5 line pairs (Ip) $/ \mathrm{mm}$ for a $14-\times$ 17-in image plate up to a theoretical maximum of $5 \mathrm{lp} / \mathrm{mm}$ on an $8-\times 10$-in size. The $\mathrm{CR}$ reader adjusts its sampling rate to the plate size to yield approximately the same number of pixels for each image (about $2,000^{2}$ pixels). This means that a body part (eg, ankle) imaged on an $8-x$ 10 - rather than $14-\times 17$-in plate would have more anatomic details as more pixels cover the area of anatomic interest. This is in contrast with a film/screen system that has the same resolution regardless of size, typically on the order of 5 to $7 \mathrm{lp} / \mathrm{mm}$ (excluding mammography). This limitation forces to use the smallest plate possible for a given body part to improve spatial resolution. Additional image quality is obtained by using the $8-\times 10$-in high-resolution 
plates having a thinner phosphor causing less blur. This allows the full $5 \mathrm{lp} / \mathrm{mm}$ to be realized with an increased patient dose similar to film/ screen extremity cassettes. The combination of superior CR contrast resolution, choosing the correct plate size for a given body part, proper use of the high-resolution plates, and image processing has resulted in overall improved image quality at the new MAMC compared with the film/screen operations at the old hospital.

Softcopy quality control operations differ from the hardcopy environment primarily in regard to image rejection and examination verification. Correct algorithm selection and collimation rules are less critical in softcopy mode. Whereas a hardcopy image with unacceptable optical density cannot be changed, the softcopy image can be windowed and leveled electronically as needed. ${ }^{33}$

Examination verifications by the qualitycontrol technologist are a linchpin operation for a successful high-volume PACS with the current level of technology. This process is combined with image reject analysis for anatomic positioning and quantum mottle. Images are then oriented correctly (eg, upright lateral chest facing to the left) based on departmental defaults or individual preferences. Finally, window and level gray-scale settings are applied by using available programmable presets. If the radiologist had been tasked with this activity, then diagnostic throughput would have been markedly compromised.

\section{Diagnostic Reporting}

Finished diagnostic reports are the final product of the radiology department. Even though everybody recognizes that timely reporting is simply good medicine, problems with reporting are nearly a universal occurrence to some degree or another. These relate to image availability for diagnosis, finding old comparison examinations, dictation, transcription, approval, and report distribution. Softcopy operations at this point at MAMC have shown that producing diagnostic reports is faster than in the film/ screen environment. In a conventional hardcopybased environment, many urgent examinations are removed from the radiology department by clinicians and not returned until later, causing a delay in official interpretation. This is elimi- nated in the PACS environment allowing unimpeded diagnosis for all examinations while image availability to all qualified users is maintained on distributed workstations. Required historical images are easily obtained from the ODJ bypassing time-consuming fileroom operations.

The radiologist assigns standard "canned" readings or types short reports for some examinations allowing real-time report completion and approval with instant availability on workstations throughout the hospital, bypassing the transcription step. Those reports requiring dictation and transcription are approved at the workstation. Reports are then printed in a central location at MAMC after approval for distribution.

\section{CLINICAL ACCEPTABILITY}

The transition toward a filmless environment has definite stages. First is a feeling of "future shock." Many of our physicians and ancillary personnel have never used computers before. This led to the next stage of user familiarization. The graphical user interface of the PACS workstations has made this step less painful. The gradual paradigm shift toward thinking in terms of digital rather than film/screen environment was the most important phase and had no definite beginning or endpoint. Development of institutional memory in using the MDIS system was the next step. Reaching the last stage of a user-integrated PACS is dependent on progressive clinical acceptance as the remainder of the software and hardware is installed.

\section{Clinician Acceptance}

Clinical acceptance of the PACS workstation has differed between clinicians and radiologists. Both groups have benefited from increased accountability and availability of images, but the primary reason for differences in acceptability is the volume of examinations viewed. Clinicians look at relatively few examinations compared with radiologists. Incompletely implemented hardware and software features have less impact on clinician's efficiency while having major impact within the radiology department. Examination availability on the workstations in areas outside of radiology is a tremendous time saver for clinicians. ${ }^{10,34}$ This is the greatest potential 
return on investment for the MDIS system. Our most conservative estimate is that 1 hour per week will be saved by the clinicians in regard to image and report availability. At least 20,000 physician hours per year can be recovered among our 400 doctors. For instance, they do not have to wait in line at the file room window for the plain films as in the past. Whereas up to $38 \%$ of recent film-based examinations were not available according to one study of a military medical center (similar to previous experience at MAMC), image availability for the MAMC MDIS System has been close to $99 \% .^{10}$ The $1 \%$ of image loss is mostly related to occasional CR interface failures and human error (eg, storing an image under the wrong patient name). The improved image accountability is the greatest reason among others for widespread acceptance of the MDIS system by clinicians.

Ambient lighting must be regulated because of the relatively lower monitor luminance currently available for the MDIS system. ${ }^{35}$ In some areas such as the emergency department (ED) the floor plan dictates locating workstations in an open work environment where control of the lighting is difficult. Whether to relocate the workstations or to design a hood of some sort to reduce ambient lighting at the monitor is still being considered. One additional workstation for the ED was placed in a nearby room where lighting can be controlled for those cases where it is definitely needed.

\section{Radiologist Acceptance}

Clinical acceptance by radiologists is different for hardcopy and softcopy operations. Acceptability of hardcopy CR images required adjustments in image size, algorithm selection, and disease detection threshold. Smaller 10- $\times 14$-in CR hardcopy format compared with the 14- $x$ 17-in film/screen images did not pose much of a problem in the transition. Quick adaptation occurred even with comparison of prior full-size films. Different possible processing algorithms for each image have taken more time for integration into clinical practice. It was necessary to recognize that if the wrong display algorithm has been used, a suboptimal image for diagnosis would be produced. A change in perception sensitivity was required in assessing interstitial markings on chest images. CR tends to accentu- ate these normal details compared with conventional film, which necessitated setting a new threshold for disease detection that was developed over several months. Improvements in image quality along with necessary transitional adaptations have made CR hardcopy clinical operations a successful replacement for traditional film/screen approaches at MAMC.

Clinical acceptance of diagnostic workstations by radiologists at MAMC have been less complete. ${ }^{10,34}$ This is not surprising because the full complement of hardware and software features are still being added at scheduled intervals. It is not enough to merely present an examination to a workstation for diagnosis in a high-volume practice. Images must be presented automatically, quickly, and efficiently.

Currently, staff radiologists are roughly divided into thirds for acceptance of the workstation at its present level of development: those who like it, those who are ambivalent in its use, and those who would rather not use it. Radiology residents are more uniform in their approach with near unanimous approval. Parenthetically, the staff radiologist who uses the workstation most frequently is in his sixties and had never used a computer before the MDIS system was installed. This illustrates how easy the user interface is to understand and learn, a consistent observation for all new users. ${ }^{10}$ Routine acceptance of softcopy reading is a function of achieving adequate diagnostic throughput in the high volume practice present at MAMC. Achieving this throughput is associated with workstation distribution, archive loading, intelligent prefetch, automatic worklists, database speed, image display speed, default display protocols, and image manipulation speed.

Emphasis to this point has been on installation of clinical workstations ( 22 to date) rather than diagnostic workstations ( 3 currently) because the level of hardware and software sophistication delivered so far supports greater success in this area. Even so, greater familiarization and improved functionality would support more softcopy reading if more diagnostic workstations were available. Primary softcopy diagnosis has been routinely used in the GI radiology section, being filmless since December 1992. Softcopy diagnosis for daytime ED and "wet" examinations has been routine since December 
1993. Remaining primary softcopy diagnostic activities at MAMC occur when prior hardcopy historical examinations cannot be found. Residents and staff are generally comfortable in using diagnostic workstations in this relatively low volume application. Furthermore, a recent MAMC pilot study reading only chest radiographs having multiple prior comparison examinations showed a $7.7 \%$ faster throughput rate for softcopy compared with hardcopy diagnosis. $^{34}$

Automatic intelligent prefetch discussed earlier is one of the most important issues for successful filmless operations of the MDIS system. ${ }^{17}$ Clinicians and radiologists both agree that this feature is one of the most essential additions to the MDIS system at MAMC. Automatic worklists currently available are organ specific (eg, chest), modality specific (eg, CT), related to referring services (eg, ED) or a combination of these. Additional "stat" and "wet" worklists are available for immediate urgent readings. However, the traditional paperdriven approach is still used because the CHCS interface to the MDIS system is not clinically available. The paper request for examination lets the radiologist know when to read an examination and is almost never in the same order as the patient names on a workstation worklist. This requires a time-consuming step of manually finding the next patient on the workstation rather than reading the next examination on the automatic worklist. The interface to CHCS should allow the use of automatic worklists necessary for high-volume softcopy operations by eliminating the paper request for examination. A more efficient electronic worklist will allow sequential softcopy reading by merely pushing a "next" button. Examinations will be read in a more timely manner with the list presented in reverse chronological order (ie, examinations waiting longest for interpretation come up first).

The MDIS system uses two networks; image and command. ${ }^{6}$ Slowdown in the Ethernet command network involving the central host processor was an unexpected problem and initially was the greatest source of clinical dissatisfaction among clinicians and radiologists. Frequent system interruptions occurred during the first few months of operation. The unpredictable length of the interruption was the most frustrating aspect. Inefficient housekeeping and worklist update functions in the MicroVAX were the cause of the problem. After several refinements, these system slowdowns are much less frequently encountered during daily operations.

Display speed of the imagery is also a critical clinical-acceptance issue. The specific solution offered by the MDIS system combines shortterm and local workstation image storage in a single device, the WSU., ${ }^{2,6}$ This offers significant advantages as long as clinical requirements can be maintained by this high-performance central file server. Initial display of a CR image takes 4 to 5 seconds from the WSU to any workstation at anytime of the day. This display speed is barely acceptable for making primary diagnosis. Subsequent display of an image from the workstation's semiconductor memory takes less than two seconds. The time to initially display a $\mathrm{CR}$ image is expected to be 2 seconds by the final implementation, making clinical acceptance more likely by radiologists. Current display rates of CT and MR examinations are approximately one image per second, which is too slow for routine clinical use.

The amount of image manipulation must be minimized to reduce the overhead of softcopy reading. ${ }^{35} \mathrm{We}$ have observed that the more image manipulation used, the more slowdown in workstation throughput occurs. ${ }^{10}$ This was our original expectation and explains why default display protocols are a cornerstone of our strategy. Presentation of new and old images on specified monitors with proper orientation at the correct window and level setting is the essence of a default display protocol. ${ }^{2,31} \mathrm{~A}$ programmable default setting for the department is used. The goal is to minimize any additional image manipulation.

Manipulation speed of images on the workstation must be near instantaneous to achieve the greatest amount of clinical acceptance. This is especially important for interactive window/ level, zoom, and magnifying functions. ${ }^{29}$ The original system specifications emphasized smooth operations of these functions during routine clinical operations. ${ }^{2}$ This has been achieved for $1-\mathrm{K}$ monitor workstations, but the speed of some functions for the $2-\mathrm{K}$ monitor workstations needs improvement for greater 
clinical acceptance. Image manipulation on the 2-K monitor is expected to improve significantly with addition of a faster image-processing board and video-display card to these workstations.

\section{CONCLUSION}

Numerous lessons have been learned in the first 2 years of operation for the MDIS system at MAMC. Incorporation of these lessons toward the last stages of the full implementation is expected to significantly improve the probability of success for "filmless" operations and acceler- ate the clinical acceptance. Future large MDIS System sites will be able to progress more rapidly to complete implementation with mature hardware and software.

\section{ACKNOWLEDGMENT}

The authors thank J. Chase, L.M. Briggs, R. Pourpasand, and $\mathrm{C}$. Stewart for their help in defining and solving many of the quality-control problems; H.D. Stigglebout for his enthusiasm and dedication in pioneering softcopy diagnosis at MAMC; MAMC's radiology staff and residents for helping to understand the radiologist acceptance issues; and D.L. Wilson and R.A. Glicksman of Loral Corporation for help on technical issues.

\section{REFERENCES}

1. Goeringer F: Medical diagnostic imaging support systems for military medicine. SPIE Proc Med Imaging 1444: 340-350, 1991

2. Medical Diagnostic Imaging Support System, Specification. Huntsville, AL, US Army Engineering Division, Solicitation No. DACA87-90-R-0058, June 22, 1990

3. Leckie RG, Walgren HN, Vincent $S$, et al: Clinical experience with teleradiology in the U.S. military. SPIE Proc Med Imaging 2165:301-311, 1994

4. Lyche DK, deTreville RE, Norton G, et al: MDIS early experience and efficacy of wide-area intercontinental teleradiology. SPIE Proc Med Imaging 2165:271-282, 1994

5. Patterson DA, Gibson G, Katz RH: A Case for Redundant Arrays of Inexpensive Disks (RAID). Report No. UCB/CSD 87/391, Berkeley, CA, University of California, December 1987.

6. Glicksman RA, Wilson DL, Perry J, et al: Architecture of a high-performance PACS based on a shared file server. SPIE Proc Med Imaging 1654:158-168, 1992

7. Stewart BK: Local area network topologies, media, and routing in: RSNA Syllabus: A Special Course in Computers for Clinical Practice and Education in Radiology. Oak Brook, IL, RSNA, 1992, pp 79-96

8. Smith DV, Smith S, Sauls F, et al: Design strategy and implementation of the medical diagnostic image support system at two large military medical centers. SPIE Proc Med Imaging 1654:148-157, 1992

9. Saarinen AO, Goodsitt M, Loop JW: The logistics of installing PACS in an existing medical center. SPIE Proc Med Imaging 1093:159-170, 1989

10. Leckie RG, Goeringer F, Smith DV, et al: Early evaluation of MDIS workstations at Madigan Army Medical Center. SPIE Proc Med Imaging 1897:336-349, 1993

11. Wilson DL: Compression in radiologic systems. SPIE Proc Med Imaging 1654:130-139, 1992

12. Ishigaki T, Sakuma S, Ikeda M, et al: Clinical evaluation of irreversible image compression: Analysis of chest imaging with computed radiography. Radiology 175 : 739-743, 1990

13. MacMahon $\mathrm{H}$, et al: Data compression: Effect of diagnostic accuracy in digital chest radiography. Radiology 178:175-179, 1991

14. Sayre J, Ho B, Boechat M, et al: Subperiosteal resorption: Effect of full-frame image compression of hand radiographs on diagnostic accuracy. Radiology 185:599-603, 1992

15. Collins CA, Lane D, Frank M, et al: Design of a receiver operating characteristic (ROC) study of 10:1 lossy image compression. SPIE Proc Med Imaging 2166:149-158, 1994

16. Meredith G, Anderson K, Wirsz E, et al: Modeling and simulation of a high performance PACS based on a shared file system architecture. SPIE Proc Med Imaging 1654:169-179, 1992

17. Wilson DL, Smith DV, Rice B: Intelligent prefetch strategies for historical images in a large PACS. SPIE Proc Med Imaging 2165:112-123, 1994

18. Bidgood WD Jr, Horii SC: PACS mini refresher course: Introduction to the ACR-NEMA DICOM standard, in RSNA Syllabus: A Special Course in Computers for Clinical Practice and Education in Radiology. pp 37-46, 1992

19. Horii SC, Bidgood WD Jr: Network and ACRNEMA protocols, in: RSNA Syllabus: A Special Course in Computers for Clinical Practice and Education in Radiology. Oak Brook, IL, RSNA, 1992, pp 97-106

20. Prior F: Specifying DICOM compliance for modality interfaces. Radiographics 13:1381-1388, 1993

21. Huang HK: Three methods of implementing a picture archiving and communication system, in: RSNA Syllabus: A Special Course in Computers for Clinical Practice and Education in Radiology, pp 47-54, 1992

22. Cawthon MA, Weiser JC, Smith DV: Application of IMACS: The military model. AAPM Summer School Proc 22:545-568, 1993

23. Taira RK, Chan KK, Stewart BK: PACS reliability issues, picture archiving and communication systems (PACS) in medicine. NATO ASI Series F 74:149-156, 1991

24. Personal communication, S.C. Horii, University of Pennsylvania, January 1994

25. Romlein J, Leckie RG, Smith S, et al: Evaluation of specific PACS equipment components-Operational and maintenance experience. SPIE Proc Med Imaging 2164:198208, 1994

26. Romlein J, Weiser JC, Sheehy MR, et al: Acceptancetesting design execution and results for large scale PACS installation. SPIE Proc Med Imaging 2165:569-581, 1994

27. Romlein J, Weiser JC, Sheehy MR, et al: Acceptance- 
testing design and execution for large-scale PACS Installation. SPIE Proc Med Imaging 1899:441-451, 1993

28. Stewart BK, Massoth RJ, Thomas SR: Mini-PACS. AAPM Summer School Proc 22:123-156, 1993

29. Haynor DR, Smith DV, Park HW, et al: Hardware and software requirements for a picture archiving and communication system's diagnostic workstations. J Digit Imaging 5:107-117, 1992

30. Weiser JC, Leckie RG, Freedman MT, et al: Significance of the Fuji AC-1 algorithms on hardcopy images. SPIE Proc Med Imaging 1897:199-203, 1993

31. Willis CE, Weiser JC, Leckie RG, et al: Optimization and quality control of computed radiography. SPIE Proc Med Imaging 2164:178-185, 1994
32. Marglin S, Rowberg A, Godwin J: Preliminary experience with portable digital imaging for intensive care radiography. J Thor Imaging 5:49-54, 1990

33. Leckie RG, Meyer C, Parker J, et al: Evaluation of traumatic lateral cervical spine computed radiography images: Quality control acceptance of images for clinical diagnosis, hardcopy versus high resolution monitors. SPIE Proc Med Imaging 1897:128-133, 1993

34. Leckie RG, Goeringer F, Smith DV, et al: The MDIS workstation-An update of performance after nearly two years of clinical use. SPIE Proc Med Imaging 2164:318-332, 1994

35. Arenson RL, Chakraborty DP, Seshadri SB: The digital imaging workstation. Radiology 176:303-315, 1990 